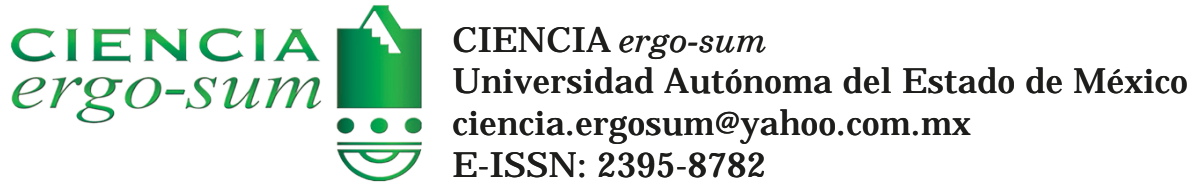

\title{
Compromiso ambiental, desempeño ambiental y desempeño: un estudio exploratorio en hoteles de tres, cuatro y cinco estrellas de cinco entidades de México
}

de Yta Castillo, Diana; Sánchez Medina, Patricia S.; Ramírez Luna, J orge A.

Compromiso ambiental, desempeño ambiental y desempeño: un estudio exploratorio en hoteles de tres, cuatro y cinco estrellas de cinco entidades de México

CIENCIA ergo-sum, vol. 26, núm. 2, julio-octubre 2019|e51

Universidad Autónoma del Estado de México, México

Esta obra está bajo una Licencia Creative Commons Atribución-NoComercial-SinDerivar 4.0 Internacional.

de Yta Castillo, D., Sánchez Medina, P. S. y Ramírez Luna, J . A. (2018). Compromiso ambiental, desempeño ambiental y desempeño: un estudio exploratorio en hoteles de tres, cuatro y cinco estrellas de cinco entidades de México. CIENCIA ergo-sum, 26(2). https://doi.org/10.30878/ces.v26n2a5 


\title{
Compromiso ambiental, desempeño ambiental y desempeño: un estudio exploratorio en hoteles de tres, cuatro y cinco estrellas de cinco entidades de México
}

Environmental commitment, environmental performance and firm performance: a study in three, four and five stars hotels of five states of Mexico

\author{
Diana de Yta Castillo \\ Universidad del Mar campus Huatulco, México \\ de_yta@yahoo.com \\ Patricia S. Sánchez Medina \\ Instituto Politécnico Nacional (CIIDIR-IPN Unidad Oaxaca), México \\ psanchez@ipn.mx \\ Jorge A. Ramirez Luna \\ Universidad del Mar campus Huatulco, México \\ jramirez@huatulco.umar.mx
}

Recepción: 04 de octubre de 2017

Aprobación: 04 de mayo de 2018

\section{RESUMEN}

Se analiza la relación entre el compromiso ambiental, el desempeño ambiental y el desempeño en hoteles de tres, cuatro y cinco estrellas de cinco entidades de México. Esta investigación es cuantitativa, transversal y exploratoria. Como técnica de recolección de datos, se aplicó un cuestionario a 55 hoteles. Para probar las hipótesis, se emplearon correlaciones bivariadas de Pearson. Los resultados muestran que el compromiso ambiental se relaciona positivamente con el desempeño ambiental y con el desempeño del hotel.

Palabras Clave: compromiso ambiental, desempeño, sector turístico, hoteles.

\begin{abstract}
The objective of this paper is to analyze the relationship between the environmental commitment and the environmental and firm performance of three-star, four-star and five-star hotels of selected entities in Mexico. This research is quantitative, transversal and exploratory. A survey was applied to 55 hotels as a technique of data collection. To prove this hypotheses, Pearson's bivariate co-relations were used. The results show that the environmental commitment is positively related to the environmental performance, and firm performance.
\end{abstract}

KEYWORDS: environmental commitment, firm performance, tourism sector, hotels.

\section{INTRODUCCIÓN}

En la actualidad el calentamiento global y el cambio climático son dos problemas que preocupan cada vez más a la sociedad porque afectan la economía, la salud y, en general, el bienestar de la población. Muchas actividades económicas contribuyen a acrecentar este problema mundial. En particular, el turismo es un sector que aporta aproximadamente 5\% de las emisiones globales de carbono (Organización Mundial del Turismo [OMT], 2010). Al mismo tiempo, el sector turístico es considerado uno de los sectores más vulnerables ante cambios climáticos (OMT, 2008), pero también puede verse afectado por otros factores. Según Galindo (2009), los cambios en la biodiversidad, el aumento en el nivel del mar, entre otros impactos ambientales, también pueden afectarlo negativamente. 
La actividad humana es sin duda una causa importante en la generación de estos efectos negativos. En este aspecto, las playas, selvas o el aire limpio tienden a sobreexplotarse y generalmente carecen de incentivos individuales para invertir en su mantenimiento y mejorar debido a que son bienes públicos. Este escenario revela la tragedia de los comunes de Hardin (1968) (Briassoulis, 2002). La problemática descrita también contribuye a la destrucción de los atractivos naturales, que son el motivo de visita de los turistas. Como se puede ver, el turismo y el medio ambiente tienen un vínculo estrecho; por ello, si el medio ambiente se deteriora, la viabilidad de la actividad turística está en riesgo.

Dado que en la actualidad existe mayor evidencia sobre los impactos del sector turístico en el medio ambiente, los temas ambientales en esta industria ganan progresivamente mayor atención en el mundo, lo cual ha provocado que en la última década haya un aumento en la investigación y en los debates sobre los desafíos ambientales (Aragon-Correa et al., 2015; Song et al., 2012).

Las investigaciones sobre temas ambientales en el turismo se han realizado desde los niveles macro y micro. En el nivel micro de la investigación, uno de los temas que se ha estudiado desde el punto de vista de la oferta, es el de las estrategias ambientales y desempeño económico de las empresas (Song et al., 2012). La investigación sobre medio ambiente en la empresa es crucial porque las empresas tienen un papel clave en la prevención del deterioro (De Burgos et al., 2013) debido a su potencial capacidad de innovación, experiencia comercial y tecnológica y al alcance de sus operaciones (Aragon-Correa et al., 2015).

En particular, los hoteles son el ente más representativo de la empresa turística (Vargas et al., 2011) y constituyen un elemento clave de la cadena de suministro de la actividad turística (Erdogan y Baris, 2007). Aunado a lo anterior, el sector alojamiento contribuye con $20 \%$ de las emisiones globales de carbono generadas por el sector turístico y consume cantidades importantes de agua, energía y recursos no duraderos (OMT, 2008; Teng et al., 2012).

Por otro lado, la gestión ambiental empresarial es una disciplina joven aún y, en consecuencia, en la literatura académica todavía no existe una definición acordada del objeto de investigación (Cramer, 1998). Una muestra de ello es que en algunos estudios se han usado diferentes variables sustitutas para medir el compromiso ambiental corporativo (Wahba, 2008). Para De Burgos et al. (2013), la protección ambiental es un constructo complejo y se relaciona con una variedad de conceptos como responsabilidad ambiental, gestión ambiental, desempeño ambiental, estrategia ambiental y proactividad ambiental. Estos conceptos muchas veces compiten entre sí y se superponen. Derivado de lo anterior, se genera un grado de confusión respecto a la terminología usada e incluso en cuanto al significado actual de las relaciones entre estos conceptos.

En este orden de ideas, para Yang et al. (2011), la buena investigación requiere rigor, relevancia y claridad. Para conseguir esto es necesario que la investigación sobre compromiso ambiental en la empresa ofrezca claridad sobre los diferentes constructos que se utilizan para referirse a la protección ambiental corporativa.

Para medir la protección ambiental de la empresa, en estudios de la literatura académica han empleado compromiso ambiental y desempeño ambiental sin hacer una clara diferencia entre estos términos. Sin embargo, cada uno de estos conceptos hace referencia a características ambientales diferentes y su influencia en el desempeño del negocio no es necesariamente el mismo (De Burgos et al., 2013). El compromiso ambiental es el medio que se emplea para alcanzar el desempeño ambiental (De Burgos y Céspedes, 2001; Johnstone y Labonne, 2009). Para Yang et al. (2011), se construye claridad al medir lo que se necesita medir. En este sentido, este artículo trata de contribuir a la literatura académica al establecer una distinción evidente entre compromiso ambiental y desempeño ambiental, para después relacionar este último con el desempeño de la empresa.

En literatura académica es posible hallar estudios que muestran una relación positiva entre el desempeño ambiental y el desempeño económico (Al-Tuwaijri et al., 2004; De Burgos y Céspedes, 2001; Russo y Fouts, 1997); sin embargo, el compromiso ambiental no necesariamente conduce a un mayor desempeño económico (García y Armas, 2007). Por otro lado, se pueden encontrar estudios que relacionan el compromiso ambiental con el desempeño de la empresa (Álvarez et al., 2001; Claver et al., 2007a; Klassen y McLauglin, 1996; Le et al., 2006) 
y otros que miden la influencia del desempeño ambiental en el desempeño empresarial (Oreja y Armas, 2012). Pero existen pocos estudios que analicen la relación del compromiso ambiental con el desempeño ambiental y de la empresa (De Burgos y Céspedes, 2001; López-Gamero et al., 2009; Molina-Azorín et al., 2009b).

Los estudios que han abordado la relación entre la respuesta ambiental de la empresa y el desempeño financiero se han enfocado en empresas manufactureras principalmente (Carmona et al., 2004). Este escenario ha generado que el sector servicios haya provocado menor atención (Álvarez et al., 2001), a pesar de que las empresas de este sector, en general, han sido señaladas como las destructoras silenciosas del medio ambiente (Hutchinson, 1996). Por lo tanto, se requiere más investigación que aborde el vínculo entre variables ambientales y desempeño empresarial en dicho sector y, en particular, en la industria hotelera (Claver et al., 2007a).

En los países en vías de desarrollo es necesario investigar sobre estos temas debido a que las iniciativas ambientales voluntarias también comienzan a ser usadas como instrumentos de política ambiental basados en incentivos (Rivera, 2002). Además, en estos países las regulaciones ambientales podrían ser laxas (Park y Kim, 2014); por este motivo, las empresas probablemente opten por involucrarse en prácticas de compromiso ambiental para mejorar sus estándares de sustentabilidad.

En el estado de Quintana Roo, México, se encuentra 40\% de las empresas de México que tiene la certificación EarthCheck (Barreto, 2015), que es una certificación para la industria del turismo que da cuenta del rendimiento ambiental y social de los entes evaluados (EarthCheck, 2016). Para este estudio, se eligió a Quintana Roo como una de las cinco entidades federativas para levantar la encuesta debido a que el entorno parecía favorable para hallar hoteles que realicen prácticas de compromiso ambiental.

En el país el nivel de implementación de prácticas ambientales en el sector hotelero es desconocido (Sánchez-Medina et al., 2016). Por lo tanto, se puede inferir que son escasas las investigaciones que estudien la relación entre el compromiso ambiental y el desempeño de los hoteles. Asimismo, la regulación ambiental en el país dirigida a los hoteles establece lo mínimo que estas empresas deben cumplir en materia ambiental (Muñoz et al., 2005). Derivado de lo anterior, este estudio trata de contribuir en llenar este vacío de la literatura académica y, por ello, su objetivo es analizar la relación entre el compromiso ambiental, el desempeño ambiental y el desempeño en hoteles de tres, cuatro y cinco estrellas de cinco entidades seleccionadas de México.

\section{Revisión de la literatura}

\section{1. Compromiso ambiental y desempeño ambiental}

La literatura de gestión ambiental ha mostrado que adoptar prácticas ambientales coadyuva a mejorar el desempeño ambiental de las empresas (Annandale et al., 2004; Aragón, 1998; Carmona et al., 2004; Zhu y Sarkis, 2004).

Para López-Gamero et al. (2009), el desempeño ambiental se relaciona con las dos formas complementarias de actividades de gestión ambiental que existen: control de la contaminación y prevención de la contaminación. Sin embargo, deberían tener un mayor impacto en el desempeño ambiental en comparación con las actividades de control de la contaminación. Las prácticas ambientales de prevención de la contaminación implican reducir, cambiar o prevenir las emisiones y descargas a través de $a$ ) una mejor gestión interna, $b$ ) sustitución de materiales, $c$ ) reciclaje o $d$ ) por medio de la innovación del proceso (Hart, 1995); de este modo, es factible que este tipo de compromiso ambiental pueda contribuir a un menor desperdicio de recursos, lo cual significaría que se usen de una forma más eficiente y productiva (Hart, 1997).

En este sentido, Álvarez et al. (2001) y Klassen y Whybark (1999) coinciden en que el compromiso ambiental preventivo permite usar las materias primas, energía y agua de una forma más eficiente, lo cual contribuye a mejorar el desempeño ambiental de la empresa al reducir o eliminar la generación de contaminantes y al disminuir el consumo de recursos. 
En el sector hotelero existen investigaciones empíricas que han abordado la relación entre compromiso ambiental y desempeño ambiental. Por ejemplo, Chan y Lam (2003), en su estudio de caso, encontraron que las prácticas ambientales de un hotel de Hong Kong conducen a mejorar la eficiencia operacional a través del ahorro de energía y agua. Por su parte, Armas (2011) encontró que los hoteles que realizan prácticas ambientales efectivas obtienen como resultado un mejor desempeño ambiental. Molina-Azorín et al. (2015) hallaron que el compromiso ambiental permite a los hoteles mejorar su desempeño ambiental a través de la reducción de impactos ambientales.

Por lo señalado en párrafos anteriores, se establece la siguiente hipótesis:

$\mathrm{H1}$ : El compromiso ambiental se relaciona directa y positivamente con el desempeño ambiental en hoteles de tres, cuatro y cinco estrellas de entidades seleccionadas de México.

\section{DESEMPEÑO AMBIENTAL Y DESEMPEÑO}

En la literatura académica se encuentran estudios que relacionan el desempeño ambiental con el desempeño de la empresa (Klassen y Whybark, 1999; Menguc et al., 2010; Wagner, 2005). Además, Albertini (2013) realizó un meta-a nálisis de 52 estudios en un periodo de 35 años sobre compromiso ambiental corporativo. La autora meta-analizó 205 coeficientes de correlación, los cuales confirman una relación positiva entre el desempeño ambiental y el desempeño financiero.

De acuerdo con López-Gamero et al. (2009), el efecto de las variables ambientales en el desempeño puede no ser inmediato. Así pues, con el fin de medir el impacto del desempeño ambiental en el desempeño de los hoteles resulta útil emplear las ventajas competitivas de costos y de diferenciación (Galdeano et al., 2008) porque permiten aislar el efecto de las prácticas ambientales en la eficiencia (Claver et al., 2007b). Para Tarí et al. (2010), la distinción entre ventaja competitiva en costos y en diferenciación es un marco teórico útil para analizar el efecto de la gestión ambiental en el desempeño empresarial.

Por un lado, el desempeño ambiental se relaciona con la ventaja competitiva en costos porque el primero implica un uso más productivo y eficiente de los recursos en la empresa. El compromiso ambiental preventivo comprende acciones que tienden a reducir el consumo de recursos, además de tareas de reutilización, reciclaje y reuso de materiales (de Yta-Castillo y Sánchez-Medina, 2017). Por ejemplo, el reciclaje impacta en el desempeño ambiental (Sroufe, 2003) y a su vez influye en los ahorros de la empresa porque evita la necesidad de adquirir nuevos insumos. Un estricto control de procesos puede contribuir a ahorrar costos porque permite mejorar la eficiencia en el uso de recursos (Porter y Van der Linde, 1995). Además, las empresas que invierten en mejorar su estrategia ambiental también evitarían accidentes ambientales (Klassen y McLaughlin, 2001). Para Hart y Milstein (2003) las empresas con una estrategia ambiental preventiva reducen los costos e incrementan las ganancias.

Por otro lado, el desempeño ambiental también puede conducir a la empresa a obtener una ventaja competitiva en diferenciación. Las prácticas ambientales de la empresa que resaltan las características ecológicas de los productos y servicios pueden captar a los consumidores verdes (Aragón y Sharma, 2003), cuya percepción le otorga mayor valor a estos productos (Galdeano et al., 2008). En otras palabras, las personas que demandan productos amigables con el medio ambiente pueden distinguir a las empresas por su nivel de desempeño ambiental.

En la actualidad existe una demanda creciente de productos verdes (Molina et al., 2015; Montabon et al., 2007). Al respecto, es factible que las empresas puedan diferenciarse de sus competidores al ofrecer productos y servicios más respetuosos con el entorno natural. La empresa con menor impacto en el medio ambiente puede enviar un mensaje positivo acerca de su preocupación por el entorno y además puede mostrar que está dispuesta a superar los estándares exigidos por la regulación ambiental (Menon y Menon, 1997).

El desempeño ambiental de la empresa crea el potencial de incrementar los precios o de establecer un precio premium por los productos y servicios, lo cual resulta en mayores ingresos (Christmann, 2000; Fraj et al., 2015). Asimismo, ser sustentable podría permitir a la empresa ganar mayor cuota de mercado y obtener 
mayores beneficios económicos como consecuencia de una opinión favorable de parte de la opinión pública debido a su compromiso con el medio ambiente (Sarkis et al., 2010).

En la literatura académica también se encuentran evidencias de que el desempeño ambiental impacta en el desempeño de los hoteles. Claver et al. (2007a) encontraron que el desempeño de la empresa crece a mayor nivel de proactividad ambiental. Pereira-Moliner et al. (2012) encontraron que la gestión ambiental impacta positivamente en el desempeño financiero, en el éxito de mercado y además en la satisfacción de los stakeholders (son individuos que se relacionan con la empresa; por ejemplo: accionistas, gobierno, clientes, entre otros). Molina-Azorín et al. (2009a) descubrieron que la gestión ambiental mejora la satisfacción de los stakeholders. Asimismo, Enz y Siguaw (1999) encontraron que las prácticas ambientales tienen un impacto positivo en la moral y orgullo de los empleados.

Tomando en cuenta señalado, se establecen las siguientes hipótesis:

H2: El desempeño ambiental se relaciona directa y positivamente con la ventaja competitiva en costos en hoteles de tres, cuatro y cinco estrellas de entidades seleccionadas de México.

H3: El desempeño ambiental se relaciona directa y positivamente con la ventaja competitiva en diferenciación en hoteles de tres, cuatro y cinco estrellas de entidades seleccionadas de México.

H4: El desempeño ambiental se relaciona directa y positivamente con el desempeño en hoteles de tres, cuatro y cinco estrellas de entidades seleccionadas de México.

\section{1. Impactos ambientales y compromiso ambiental del sector hotelero}

Las empresas del sector servicios, en general, han sido señaladas como las destructoras silenciosas del medio ambiente (Hutchinson, 1996) porque su impacto ambiental no está tan expuesto al público como el de las empresas del sector industrial. Sin embargo, las empresas del sector terciario son negocios que contaminan de forma importante. Según Armas (2011), estos negocios son responsables de la mayor parte de la degradación ambiental del mundo.

Los hoteles, como la mayoría de las empresas del sector servicios, se caracterizan por tener un impacto ambiental fragmentado en muchas actividades, lo cual provoca que tengan muchas fuentes de contaminación. En lo individual, estas operaciones generan, directa o indirectamente, pequeñas cantidades de contaminación (Armas, 2011; Carmona et al., 2004; Céspedes y de Burgos, 2004; Kirk, 1995). Sin embargo, si todas estas pequeñas operaciones se suman, la cantidad total que genera la industria hotelera se vuelve importante e impacta de manera significativa en los recursos globales (Kirk, 1995).

Dado que el impacto ambiental de los hoteles es difuso, se presenta la dificultad de regularlo y controlarlo sólo por medio de la regulación ambiental. Aunado a lo anterior, los hoteles no generan grandes cantidad de residuos peligrosos (Carmona et al., 2004; Céspedes y de Burgos, 2004). Por ello, en estas empresas las prácticas de prevención son más comunes que las de control de la contaminación (Carmona et al., 2004; Céspedes y de Burgos, 2004).

Por otra parte, el medio ambiente y el turismo están ligados debido a que la actividad turística depende de unos recursos naturales sanos y atractivos para seguir creciendo económicamente. Un medio físico en buen estado refleja mayor calidad del producto turístico (García y Armas, 2007) y del destino turístico, lo cual atrae a un mayor número de turistas, lo que a su vez puede generar mayor prosperidad económica para distintas regiones en un país. En sentido contrario, la degradación del entorno natural atenta contra la continuidad de esta actividad económica (El Dief y Font, 2010).

En la literatura académica existe suficiente evidencia de que los hoteles impactan negativamente el medio ambiente al expulsar emisiones a la atmósfera, generar aguas residuales, destruir áreas naturales para la construcción de alojamientos, consumir cantidades considerables de energía y de agua, entre otros daños (Erdogan y Baris, 2007; Fukey e Isaac, 2014; Le et al., 2006). En otras palabras, estos negocios dejan una huella ecológica en el medio natural (Bohdanowicz, 2005). 
Derivado de la regulación ambiental, la exposición al público de sus impactos ambientales y de la presión de los consumidores más conscientes en materia ambiental, muchos hoteles se han involucrado en el desarrollo de iniciativas ambientales. Como señalan Erdogan y Baris (2007), muchos gerentes de hoteles han entendido que el crecimiento económico y la sustentabilidad de largo plazo dependen de su involucramiento en estrategias ambientales. En este tenor, un gran número de hoteles toman el compromiso ambiental como una variable estratégica que podría permitirles tener éxito en el contexto competitivo actual (García y Armas, 2007; Sharma y Vredenburg, 1998).

En los hoteles las prácticas ambientales que predominan son aquellas que minimizan el uso de recursos y costos, introducen la compra verde, tienen bajo costo y se pueden realizar durante los procesos normales de operación de la empresa (Garay y Font, 2012; Oreja y Armas, 2012).

Los hoteles pueden optar por soluciones formales o informales (Sánchez-Medina et al., 2016). Los pequeños hoteles tienden a implementar prácticas ambientales como el compromiso y ética ambiental (Kumar, 2015), las cuales no implican una certificación formal (Sánchez-Medina et al., 2016). En cambio, los grandes hoteles son más propensos a desarrollar y usar sistemas de gestión ambiental (Henri y Journeault, 2008).

\section{2. Impactos y prácticas ambientales de los hoteles de México}

En México también existe evidencia del deterioro ambiental que genera el turismo (Luja y Saldaña, 2014; Vargas et al., 2011) y la hotelería (Vargas, 2015). En Cancún, las empresas hoteleras han ocasionado la pérdida de la diversidad biológica, además de contaminación de tierra, agua y aire que, junto con otras actividades económicas de las que se apoyan, producen una gran cantidad de desechos, erosionan el suelo y son claros ejemplos de la destrucción del paisaje y la desaparición de las condiciones de vida de las diferentes especies (Vargas, 2015).

De acuerdo con el Instituto Mexicano para la Competitividad, A. C. (2013), cada cuarto de hotel genera 407 $\mathrm{kg}$ de residuos sólidos al año, con un promedio máximo de $522 \mathrm{~kg}$ registrado en Cancún, Quintana Roo, y un mínimo de $342 \mathrm{~kg}$ en Zihuatanejo, Guerrero. En cuanto al consumo de agua por persona, la Riviera Maya ocupa el sitio más alto con $1137.58 \mathrm{~m}^{3}, 38 \%$ por encima de Cancún que, a su vez, es $110 \%$ superior al de Mazatlán, cuyo consumo es de $388 \mathrm{~m}^{3}$. El resto de los destinos turísticos tiene consumos por persona similares por debajo de los $400 \mathrm{~m}^{3}$ (IMCO, 2013). Este último dato representa un consumo mayor al promedio mundial conservador de 222 l. Esto sugiere un consumo muy elevado de agua, mayor al que los turistas hacen en sus hogares (Luja y Saldaña, 2014). La razón de este particular comportamiento radica en que los huéspedes tienden a tener un "enfoque de placer" para bañarse y usan más agua de la que emplean en casa (Kasim et al., 2014).

En el país, el nivel de implementación de prácticas ambientales en el sector hotelero es desconocido (Sánchez-Medina et al., 2016). Sin embargo, se tiene evidencia de que esta industria desarrolla prácticas ambientales. Por ejemplo, se han implementado medidas de separación de residuos y reciclaje, manejo de planes de emergencia y residuos peligrosos y cálculos de consumo de agua y energía (de Yta-Castillo y Sánchez-Medina, 2017, en prensa; Vargas, 2015), medidas de ahorro de energía y agua (Sánchez-Medina et al., 2016) e introducción de ecotecnologías (Vargas, 2015).

Se puede observar que los hoteles en México producen una importante cantidad de contaminación y consumen significativas sumas de recursos naturales, y, en sintonía con la tendencia mundial, el sector hotelero del país está llevando a cabo prácticas ambientales como parte de sus actividades diarias. De este modo, resulta de interés conocer si el compromiso ambiental en los hoteles los conduce a un mejor desempeño ambiental y, en general, a un mejor desempeño de la empresa.

\section{Método}

De acuerdo con la metodología para las ciencias sociales (Hernández et al., 2010), esta investigación es cuantitativa, transversal y exploratoria. Como técnica de recolección de datos se utilizó la encuesta; para esto, se aplicó un cuestionario a la persona que el hotel designara como la más apropiada para responder un instrumento de medición de esta 
naturaleza. Entre los puestos de los encuestados se encuentran gerentes, jefes o coordinadores de gestión ambiental y de sustentabilidad, de normatividad ambiental, de calidad, de ventas, de mantenimiento o de recursos humanos.

El instrumento está compuesto por 19 preguntas que recogieron información general del hotel y del encuestado: cuatro preguntas miden el compromiso ambiental, seis miden el desempeño ambiental, nueve miden la ventaja competitiva y seis miden el desempeño del hotel. La escala del cuestionario fue una escala Likert de 5 puntos, donde 1 es "nada" y 5 es "muchísimo". El periodo de la encuesta fue de enero a abril de 2017. La muestra quedó integrada por 55 hoteles, de los cuales 67\% es de Quintana Roo, 16\% de Oaxaca, 11\% de Puebla, 4\% de Nayarit y $2 \%$ restante de Guanajuato.

\section{1. Muestra y encuesta}

Esta investigación exploratoria buscó reunir el mayor número posible de cuestionarios contestados en cada entidad federativa seleccionada en el periodo de tiempo destinado. Sin embargo, sí se establecieron estratos con base en la categoría y entidad federativa donde se ubica el hotel. Respecto al primer criterio, se eligieron los hoteles de las categorías tres, cuatro y cinco estrellas debido a que en otros trabajos se ha elegido este tipo de hoteles (Álvarez et al., 2001; Claver et al., 2008; Pereira-Moliner et al., 2012) por la homogeneidad que presentan entre ellos (García y Armas, 2007). En cuanto al segundo criterio, se eligió al estado de Quintana Roo debido a que allí se encuentra 40\% de las empresas de México que poseen la certificación EarthCheck (Barreto, 2015), por lo que parecía un entorno propicio para encontrar hoteles que desarrollasen prácticas de compromiso ambiental. El resto de los estados se eligieron por razones de logística y de presupuesto para realizar la encuesta. En una etapa posterior, los hoteles se eligieron al azar a partir de diferentes directorios y de la conveniencia que representaba su ubicación en cada una de los destinos.

En una primera fase de la encuesta se realizó una entrevista no estructurada con un grupo de gerentes y dueños con la finalidad de conocer el tipo de estrategias ambientales que estaban desarrollando en sus hoteles. En una segunda fase se llevó a cabo una prueba piloto con el objetivo de corregir y mejorar las preguntas y las escalas de opinión para establecer el instrumento final que sería aplicado. De la encuesta final, se obtuvieron 55 hoteles de tres, cuatro y cinco estrellas de cinco estados de la República Mexicana. Se visitó personalmente a 95\% de los hoteles encuestados; a 5\% restante se le envió los cuestionarios por medio de correo electrónico. De los hoteles visitados en persona, en $60 \%$ de los casos el cuestionario se aplicó personalmente y en $40 \%$ restante, por razones de conveniencia, se dejó el instrumento de medición a los gerentes y dueños que despúes devolvieron por correo electrónico cuando estuvieron contestados.

\section{2. Medición de las variables}

a) Compromiso ambiental: con base en Álvarez et al. (2001), De Burgos et al. (2013) y López-Gamero et al. (2008), son las actividades que el hotel realiza para reducir la producción de contaminantes y el uso de recursos naturales que implican sus operaciones.

b) Desempeño ambiental: con base en De Burgos y Céspedes (2001), Johnstone y Labonne (2009) y De Burgos et al. (2013), es el resultado de las actividades que el hotel realiza para reducir la producción de contaminación y el uso de recursos naturales que implican sus operaciones.

c) Ventaja competitiva: con base en Banerjee et al. (2003), se define como la posición competitiva del hotel en donde experimenta una disminución de costos y logra diferenciar sus productos y servicios de sus competidores debido a su desempeño ambiental.

d) Desempeño de la empresa: con base en Venkatraman y Ramanujam (1986) y Claver-Cortés et al. (2008), son los resultados financieros y operacionales que el hotel experimenta derivado de los esfuerzos de compromiso ambiental. 
Para medir el compromiso ambiental se emplearon cuatro preguntas de la literatura de gestión ambiental (Álvarez et al., 2001; De Burgos et al., 2013; López-Gamero et al., 2008). Para medir el desempeño ambiental, se utilizaron seis preguntas de Molina-Azorín et al. (2015). Con el objetivo de medir la ventaja competitiva en costos se emplearon cinco preguntas de la literatura académica de gestión ambiental (Banerjee et al., 2003; Christmann, 2000; Leonidou et al., 2013; Shrivastava, 1995). Para medir una ventaja competitiva en diferenciación se utilizaron cuatro preguntas de la literatura académica (Leonidou et al., 2013; López-Gamero et al., 2010; Maas et al., 2014). Finalmente, para medir la variable desempeño del hotel se emplearon seis preguntas de Molina-Azorín et al. (2009a) (tabla 1).

TABLA 1

Operacionalización de las variables del estudio

\begin{tabular}{|c|c|c|c|}
\hline Variable & Autor & Preguntas & Escala \\
\hline \multirow{4}{*}{ Compromiso ambiental } & Ál & Medidas de ahorro de agua & \multirow{4}{*}{$\begin{array}{l}\text { Tipo Likert de } 5 \\
\text { puntos }\end{array}$} \\
\hline & Aivarez el al. $(2001)$ & Medidas de ahorro de energía & \\
\hline & De Burgos et al. (2013) & Reúso/reciclaje de productos & \\
\hline & $\begin{array}{l}\text { López-Gamero et al. } \\
\text { (2008) }\end{array}$ & $\begin{array}{l}\text { Respeto y promoción de la vegetación } \\
\text { autóctona en jardínes y áreas verdes (medida } \\
\text { de ahorro de agua) }\end{array}$ & \\
\hline \multirow{6}{*}{ Desempeño ambiental } & \multirow{6}{*}{$\begin{array}{l}\text { Molina-Azorín et al. } \\
\text { (2015) }\end{array}$} & Menor consumo de agua & \multirow{6}{*}{$\begin{array}{l}\text { Tipo Likert de } \\
\text { puntos }\end{array}$} \\
\hline & & Menor consumo de energía & \\
\hline & & Menor uso de recursos no renovables & \\
\hline & & Menor uso de materias primas tóxicas & \\
\hline & & Menor cantidad de desperdicios sólidos & \\
\hline & & Menor cantidad de descargas & \\
\hline \multirow{5}{*}{$\begin{array}{l}\text { Ventaja competitiva en } \\
\text { costos }\end{array}$} & Banerjee et al. (2003) & Obtención de sustanciales ventajas en costos & \multirow{5}{*}{$\begin{array}{l}\text { Tipo Likert de } 5 \\
\text { puntos }\end{array}$} \\
\hline & Christmann (2000) & $\begin{array}{l}\text { Menores costos derivados de la regulación } \\
\text { gubernamental en el tema ambiental }\end{array}$ & \\
\hline & & Mejora en la posición en costos & \\
\hline & Leonidou et al. (2013) & $\begin{array}{l}\text { Ahorros significativos en costos por el } \\
\text { mejoramiento de la calidad en los servicios }\end{array}$ & \\
\hline & Shrivastava (1995) & $\begin{array}{l}\text { Ahorros en costos derivados de usar menos } \\
\text { recursos naturales y energía en las actividades }\end{array}$ & \\
\hline \multirow{3}{*}{$\begin{array}{l}\text { Ventaja competitiva en } \\
\text { diferenciación }\end{array}$} & Leonidou et al. (2013) & Ser líder en el mercado & \multirow{3}{*}{$\begin{array}{l}\text { Tipo Likert de } \\
\text { puntos }\end{array}$} \\
\hline & $\begin{array}{l}\text { López-Gamero et al. } \\
\text { (2010) }\end{array}$ & $\begin{array}{l}\text { Mejora en la imagen de la marca } \\
\text { Mayor credibilidad en las relaciones } \\
\text { comerciales }\end{array}$ & \\
\hline & Maas et al. (2014) & Mejora en la reputación de la empresa & \\
\hline \multirow{6}{*}{$\begin{array}{l}\text { Desempeño de la } \\
\text { empresa }\end{array}$} & \multirow{6}{*}{$\begin{array}{l}\text { Molina-Azorín et al. } \\
\text { (2009a) }\end{array}$} & Tasa de ocupación por cuarto & \multirow{6}{*}{$\begin{array}{l}\text { Tipo Likert de } \\
\text { puntos }\end{array}$} \\
\hline & & Promedio de ventas & \\
\hline & & Ingreso por cuarto & \\
\hline & & Nivel de satisfacción del empleado & \\
\hline & & Nivel de satisfacción de la gerencia & \\
\hline & & Nivel de satisfacción del dueño y accionistas & \\
\hline
\end{tabular}

Fuente: elaboración propia con base en los estudios señalados. 


\section{AnÁlisis y discusión de RESUltados}

El análisis de los datos se realizó con el programa SPSS versión 17. Para la prueba de hipótesis se realizaron correlaciones bivariadas de Pearson. La correlación bivariada de Pearson muestra que el compromiso ambiental tiene una relación positiva y significativa $(r=0.482, p \leq 0.01)$ con el desempeño ambiental. Estos resultados permiten aceptar la hipótesis 1 (cuadro 1). En los hoteles de la muestra, las medidas de ahorro de agua y de energía y el reúso reciclaje les permiten mejorar su desempeño ambiental a través de un uso más eficiente de sus recursos.

La industria hotelera es considerada un sector intensivo en el uso de energía y agua (Kasim, 2007; Xuchao et al., 2010). Asimismo, en un hotel existe una gran cantidad de operaciones en donde se emplea agua y energía, y además se usa también con fines recreativos. En ese sentido, hay evidencia de que los turistas hacen en promedio un consumo mayor de agua en comparación con el consumo que hacen en sus hogares (Luja y Saldaña, 2014). En relación al consumo de energía, es bien conocido que las operaciones de los hoteles se llevan a cabo durante las 24 horas del día, lo cual significa que un turista consume una cantidad de energía mayor a la que usaría en su hogar. Con este antecedente, parece natural encontrar que los hoteles estudiados desarrollen prácticas ambientales que les permitan minimizar la utilización de recursos y de esta manera mejorar la eficiencia en su uso.

Los resultados de este trabajo coinciden con los de López-Gamero et al. (2009) y Molina et al. (2015), quienes analizaron la relación gestión ambiental-desempeño ambiental en el ámbito hotelero. Estos autores distinguen entre gestión ambiental y desempeño ambiental. La primera incluye medidas que disminuyen el uso de recursos naturales y que promueven una reducción de los desperdicios y, por su parte, el desempeño ambiental se refiere a los resultados derivados de estas prácticas ambientales. En ambos estudios se encontró una relación positiva entre gestión ambiental y desempeño ambiental (tabla 2).

\section{CUADRO 1}

Correlación bivariada entre las variables del estudio

\begin{tabular}{|c|c|c|c|c|c|c|}
\hline Variable & $\mathrm{N}$ & 1 & 2 & 3 & 4 & 5 \\
\hline $\begin{array}{l}\text { Compromiso } \\
\text { ambiental }\end{array}$ & 55 & 1 & $0.482 * *$ & $0.416 * *$ & 0.110 & $0.385 * *$ \\
\hline $\begin{array}{l}\text { Desempeño } \\
\text { ambiental }\end{array}$ & 55 & & 1 & $0.360 * *$ & 0.111 & 0.204 \\
\hline $\begin{array}{l}\text { Ventaja competitiva } \\
\text { en costos }\end{array}$ & 55 & & & 1 & 0.122 & $0.306 *$ \\
\hline $\begin{array}{l}\text { Ventaja competitiva } \\
\text { en diferenciación }\end{array}$ & 55 & & & & 1 & -0.132 \\
\hline $\begin{array}{l}\text { Desempeño de la } \\
\text { empresa }\end{array}$ & 55 & & & & & 1 \\
\hline
\end{tabular}

Fuente: elaboración propia con base en los datos obtenidos de la encuesta.

Nota: ${ }^{* *} p \leq 0.01 ;{ }^{*} p<0.05$

La correlación bivariada de Pearson muestra que el desempeño ambiental tiene una relación positiva y significativa $(r=0.360, p \leq 0.01)$ con la ventaja competitiva en costos. Estos resultados permiten aprobar la hipótesis 2 (cuadro 1). En la muestra de hoteles estudiados, el menor consumo recursos naturales y de energía y la menor cantidad de desperdicios y descargas permiten alcanzar una ventaja en costos. Durante las entrevistas, algunos encuestados señalaron que ahorrar en sus costos es el principal motivo para llevar a cabo prácticas ambientales.

En los hoteles, como en otras empresas del sector turístico, las actividades sustentables que todavía dominan son las que disminuyen costos (Garay y Font, 2012). Estas prácticas ambientales tienen mayor probabilidad de tener éxito en su implementación en comparación con el resto (por ejemplo las estrategias ambientales para diferenciar la empresa del resto de los competidores) (Jones et al., 2014 en Walsh y Dodds, 2017). Además, este tipo de prácticas no requiere grandes inversiones financieras, pero provee un beneficio financiero inmediato (Bagur-Femenias et al., 2013). 
En el marco de los estudios en el sector hotelero, estos resultados coinciden con Molina et al. (2015) al hallar resultados similares al indicar que el desempeño ambiental contribuye a alcanzar una ventaja competitiva en costos, pero no concuerdan con López-Gamero et al. (2009), quienes hallaron una relación negativa y no significativa entre estas variables (tabla 2).

La correlación bivariada de Pearson muestra que el desempeño ambiental tiene una relación positiva y no significativa $(r=0.204, p \geq 0.05)$ con el desempeño de la empresa. Estos resultados no permiten aprobar totalmente la hipótesis 4 (cuadro 1). Este resultado implica que los gerentes de los hoteles consideran que el desempeño ambiental sí permite obtener un ahorro en costos, pero no les posibilita que obtengan mayores ganancias en términos de dinero. Esta situación puede deberse a que los gerentes consideran que algunas inversiones ambientales son difíciles de recuperar en el corto plazo y que nada más a largo plazo pueden verse los beneficios económicos cuando dichas inversiones hayan sido amortizadas (Fraj et al., 2009).

Otra razón para explicar por qué los hoteleros no perciben un incremento en el desempeño del hotel puede encontrarse en el hecho de que para tener mayores niveles de ventas deberían incrementar sus precios por cuarto. Sin embargo, de hacerlo, quizá correrían el riesgo de perder a parte de su clientela porque para algunos consumidores el precio sigue siendo una variable importante al elegir un hotel (Fukey e Isaac, 2014). Asimismo, para alcanzar una mayor tasa de ocupación por tener mejor desempeño ambiental sería necesario tener un mayor número de consumidores más conscientes ambientalmente que prefieran las iniciativas verdes por encima de la comodidad y el confort del hotel. En México es probable que el grupo de consumidores con estas características esté aún en crecimiento. En general, el tema de la sustentabilidad en la sociedad de países en vías de desarrollo es un asunto que carece de la importancia que se le otorga en algunos países desarrollados (Park y Kim, 2014).

De los estudios en la hotelería, este resultado concuerda con los hallazgos de Álvarez et al. (2001), quienes encontraron que el desempeño financiero medido en términos de ganancias en el último año, ganancias en los últimos tres años y tasa de ocupación tiene una leve relación con la gestión ambiental. Además, este resultado coincide parcialmente con Armas (2011) quien halló que el desempeño ambiental tiene un efecto positivo en las ganancias y ventas de los hoteles de España. Por su lado, los hallazgos del trabajo no coinciden con Rivera (2002), quien encontró que un mayor desempeño ambiental no está correlacionado significativamente con mayores niveles de ocupación, los cuales podrían incrementar las ganancias de los hoteles en términos de dinero.

Los resultados de este trabajo tampoco concuerdan con Walsh y Dodds (2017), quienes encontraron que las estrategias como medidas de conservación de energía y agua y actividades de gestión de los residuos no proveen un efecto positivo en el éxito económico de los hoteles de Estados Unidos.

Por otra parte, el resultado de esta investigación concuerda también de manera parcial con los resultados de Klassen y McLauglin (1996), quienes en empresas del sector manufacturero, servicios eléctricos y de extracción de petróleo y de gas encontraron que aquellas con un alto desempeño ambiental experimentaron un aumento en los rendimientos de sus acciones de mercado. Por otro lado, Wagner et al. (2002) en empresas europeas de la industria manufacturera del papel hallaron una relación negativa entre desempeño ambiental y desempeño económico (tabla 2).

Un hallazgo adicional es que existe una relación positiva y significativa $(r=0.385, p \leq 0.01)$ entre el compromiso ambiental y el desempeño de la empresa. Este resultado difiere del de García y Armas (2007) para quienes el compromiso ambiental no necesariamente conduce a un mayor desempeño económico en hoteles de España. Este resultado podría deberse a que los hoteleros perciben que las prácticas para disminuir el consumo de energía y recursos naturales impactan de manera directa en el desempeño del negocio porque les permite ahorrar.

Como puede notarse, los resultados muestran que las hipótesis $\mathrm{H} 1$ y H2 se aceptan y las hipótesis $\mathrm{H} 3$ y $\mathrm{H} 4$ se aceptan de modo parcial. Lo anterior significa que el compromiso ambiental se relaciona positivamente con el desempeño ambiental, y este último con la ventaja en costos, ventaja en diferenciación y el desempeño del hotel, aunque con estas dos últimas variables lo hace de manera no significativa. 
TABLA 2

Comparación de los resultados de este estudio con hallazgos de otras investigaciones de naturaleza semejante

\begin{tabular}{|c|c|c|c|c|}
\hline & \multicolumn{2}{|c|}{ Sector hotelero } & \multicolumn{2}{|c|}{ Otros sectores económicos } \\
\hline $\begin{array}{l}\text { Resultados de relaciones entre } \\
\text { variables }\end{array}$ & Similares & Diferentes & Similares & Diferentes \\
\hline Compromiso ambiental- & & & $\begin{array}{l}\text { Annandale et } \\
\text { al.(2004) }\end{array}$ & \\
\hline aesempeno amoiental & Molina-Azorín et al. (2015) & & Zhu y Sarkis (2004) & \\
\hline $\begin{array}{l}\text { Desempeño ambiental-ventaja } \\
\text { competitiva en costos }\end{array}$ & Molina-Azorín et al. (2015) & $\begin{array}{l}\text { López-Gamero et } \\
\text { al. (2009) }\end{array}$ & & \\
\hline Desempeño ambiental-ventaja & López-Gamero et al.(2009) & & & \\
\hline competitiva en diferenciación & Molina-Azorín et al. (2015 & & & \\
\hline $\begin{array}{l}\text { Desempeño ambiental- } \\
\text { desempeño }\end{array}$ & Armas (2011) & $\begin{array}{l}\text { Walsh y Dodds } \\
\text { (2017) }\end{array}$ & $\begin{array}{l}\text { Klassen y } \\
\text { McLaughlin (1996) }\end{array}$ & $\begin{array}{l}\text { Wagner et } \\
\text { al. (2002) }\end{array}$ \\
\hline
\end{tabular}

Fuente: elaboración propia.

\section{Conclusiones}

En la actualidad los teóricos organizacionales muestran un incesante interés por estudiar los desafíos que enfrentan las empresas en el tema ambiental. Estos estudios tratan de exponer alternativas de solución que permitan a las empresas remediar en cierto grado los daños provocados al medio ambiente. En este trabajo exploratorio se analizó la relación entre compromiso ambiental, desempeño ambiental y desempeño en hoteles de tres, cuatro y cinco estrellas de cinco entidades de México. De este análisis, se obtuvo que el compromiso ambiental se relaciona directa y positivamente con el desempeño ambiental de los hoteles y que el desempeño ambiental se relaciona directa y positivamente con la ventaja competitiva en costos. Asimismo, se halló que el desempeño ambiental se relaciona positiva y no significativamente con la ventaja competitiva en diferenciación y con el desempeño de los hoteles. De esta manera, estos resultados permiten contribuir a la literatura desde diversas vertientes:

a) Se estudia el compromiso ambiental como una variable distinta al desempeño ambiental y se muestra que son conceptos que hacen referencia a características ambientales diferentes y que además guardan una relación directa y positiva. En este sentido, se contribuye con la literatura académica que señala al compromiso ambiental como una variable distinta al desempeño ambiental (De Burgos y Céspedes, 2001; De Burgos et al., 2013; Johnstone y Labonne, 2009). Además, se puede ver que el compromiso ambiental es el medio para conseguir el desempeño ambiental, lo cual coincide con De Burgos y Céspedes (2001) y Johnstone y Labonne (2009).

b) Una aportación más radica en analizar la relación entre compromiso ambiental con el desempeño ambiental y de la empresa, con lo cual se contribuye a la literatura académica que indica que aún existen pocas investigaciones que aborden la relación entre estas variables en un solo estudio (De Burgos y Céspedes, 2001; López et al., 2009; Molina-Azorín et al., 2009b).

c) Se estudia la relación entre desempeño ambiental y ventaja competitiva debido a que el efecto del primero en el desempeño del hotel puede no ser inmediato y más bien en un principio reflejarse en una mayor ventaja competitiva, ya sea en costos y en diferenciación. Se halló que el desempeño ambiental se relaciona significativamente con la ventaja en costos, pero no con la ventaja en diferenciación. Este resultado coincide con la literatura académica que señala que las prácticas ambientales más atractivas para los hoteleros son las que reducen sus costos (Garay y Font, 2012) y que las iniciativas para diferenciar al hotel tienen menos probabilidad de ser exitosas (Jones et al., 2014 en Walsh y Dodds, 2017). 
d) Asimismo, se analiza la relación entre desempeño ambiental y desempeño del hotel encontrándose que se relacionan de forma no significativa, lo cual concuerda con López-Gamero et al. (2009), para quienes el efecto de las variables ambientales en el desempeño no necesariamente es inmediato. Los hoteleros de la muestra perciben que al realizar iniciativas ambientales consiguen ahorros, pero que las estrategias ambientales no son suficientes para mejorar el desempeño de sus negocios. Cabe destacar que se esperaba una relación significativa entre desempeño ambiental y desempeño del hotel, pero no se logró con la muestra del estudio, a diferencia de la relación compromiso ambiental-desempeño que sí resultó significativa. Este hallazgo concuerda con De Burgos et al. (2013), quienes manifiestan que la influencia de cada uno de estos conceptos en el desempeño financiero no es necesariamente el mismo.

e) Se estudia un sector al que se le ha brindado poca atención en temas ambientales, pero que se ha comprobado impacta de manera importante el ambiente natural. En los hoteles el compromiso ambiental se manifiesta sobre todo en prácticas para prevenir la contaminación, más que nada debido a que su generación se presenta de manera difusa. Aunado a lo anterior, la regulación ambiental es laxa para este sector; quizá por ello los hoteles de la muestra optan por iniciativas ambientales voluntarias para conservar el medio ambiente. Debido a esto, resulta importante la investigación del compromiso ambiental de los hoteles y su relación con el desempeño ambiental y desempeño porque permite mostrar los beneficios económicos de proteger el medio ambiente. La legitimidad económica del compromiso ambiental empresarial podría incentivar a la industria turística en general a trabajar en favor del medio ambiente.

\section{LIMITACIONES DEL ESTUDIO Y LÍNEAS DE INVESTIGACIÓN A DESARROLLAR}

El trabajo tiene algunas limitaciones, lo cual da lugar a que se realicen más investigaciones a futuro. El tamaño de la muestra es una limitante, ya que sólo son 55 hoteles de cinco estados de la República Mexicana. Para futuros estudios, se recomendaría incluir un mayor número de empresas hoteleras y un mayor número de entidades. En este sentido, es necesario mencionar que la experiencia de encuesta muestra que en México es indispensable fomentar una cultura empresarial que promueva el comportamiento de información del negocio para realizar investigaciones académicas. De superar esta limitante, se podría estar en posibilidad de obtener una muestra representativa de las empresas hoteleras.

$\mathrm{Al}$ mismo tiempo, es recomendable incluir a hoteles de categorías inferiores a las referidas, puesto que estos negocios también desarrollan actividades de gestión ambiental, aunque de manera menos formal en comparación con las empresas de categoría superior. Por estas circunstancias, se sugiere realizar estudios sobre compromiso ambiental, desempeño ambiental y desempeño en hoteles tanto de naturaleza más profesional que familiar y en aquellos con una naturaleza más familiar que profesional.

Finalmente, una limitante más es que un análisis de correlación se emplea para conocer la fuerza y el sentido con que se relacionan dos variables, pero no permite conocer el efecto de una variable independiente sobre una dependiente. Para investigaciones futuras en torno a este tema, entonces, se sugiere utilizar un análisis de regresión con las variables del estudio.

\section{Análisis Prospectivo}

En el contexto actual de los problemas ambientales que enfrenta la humanidad, este estudio pretende hacer notar que los beneficios económicos derivados de las iniciativas ambientales voluntarias de las empresas pueden ser un motivo importante para estimular a los empresarios a realizar acciones de conservación del medio ambiente.

En particular, este trabajo estudia a empresas del sector turístico, el cual es uno de los sectores económicos con mayor empuje económico hoy en día, y del cual se preveé un crecimiento considerable en un futuro. En sus previsiones de largo plazo, la OMT pronostica que la llegada de turistas internacionales crezca un $3.3 \%$ al año 
entre 2010 y 2030 hasta llegar a los 1800 millones en 2030 (OMT, 2016). Con este panorama, ante el desarrollo de esta actividad económica, es necesario hacer visible si los hoteles pueden obtener resultados económicos tangibles al realizar actividades de gestión ambiental. Es posible que los hoteles tengan mayor disposición a trabajar en favor del medio ambiente si las consecuencias son benéficas para ellos.

Aunado a lo presentado, desarrollar prácticas ambientales en los hoteles contribuye a la propia supervivencia del sector turístico, ya que el medio ambiente y el turismo están estrechamente relacionados. La actividad turística contribuye al calentamiento global y al cambio climático, problemas ambientales que a la vez ponen en riesgo su supervivencia.

Además, debido a que la regulación ambiental en este sector es laxa, es indispensable mostrar los incentivos económicos para que los empresarios del sector hotelero desarrollen prácticas de gestión ambiental.

\section{Agradecimientos}

Este estudio forma parte del proyecto de investigación denominado "Gestión de la calidad, gestión ambiental y desempeño empresarial: un estudio en la industria hotelera mexicana”. Los autores de la Universidad del Mar agradecen al Programa para el Desarrollo Profesional Docente para el tipo superior (PRODEP) por el financimiento otorgado para realizar esta investigación.

\section{Referencias}

Albertini, E. (2013). Does environmental management improve financial performance? A meta analytical review. Organization Environment, 26(4), 431-457.

Al-Tuwaijri, S. A., Christensen, T. E., \& Hughes II, K. E. (2004). The relations among environmental disclosure, environmental performance, and economic performance: A simultaneous equations approach. Accounting, Organizations and Society, 29, 447-471.

Álvarez, M., De Burgos, J., \& Céspedes, J. (2001). An analysis of environmental management, organizational context and performance of Spanish hotels. Omega, 29(6), 457-471.

Annandale, D., Morrison, A., \& Bouma, G. (2004). The impact of voluntary environmental protection instruments on company environmental performance. Business Strategy and the Environment, 13(1), 1-12.

Aragón, J. (1998). Strategic proactivity and firm approach to the natural environment. The Academy of Management Journal, $41(5), 556-567$.

Aragón, J., \& Sharma, J. (2003). A contingent resource-based view of proactive corporate environmental strategy. Academy of Management Review, 28(1), 71-88.

Aragon-Correa, J., Martin-Tapia, I., \& De la Torre-Ruiz, J. (2015). Sustainability issues and hospitality and tourism firms' strategies: Analytical review and future directions. International Journal of Contemporary Hospitality Management, 27(3), 498-522.

Armas, Y. (2011). Determinants of environmental management and implications for firms' economic performance. Journal of Environmental Planning and Management, 54(8), 1077-1106.

Bagur-Femenias, L., Llach, J., \& Alonso-Almeida, M. (2013). Is the adoption of environmental practices a strategical decision for small service companies? An empirical approach. Management Decision, 51(1), 41-62.

Banerjee, S. B., Iyer, E. S., \& Kashyap, R. K. (2003). Corporate environmentalism: Antecedents and influence of industry type. Journal of Marketing, 67(2), 106-122.

Barreto, A. (2015). El 40\% de las empresas EarthCheck de México, en Q. Roo. Novedades en Quintana Roo. Disponible en http://sipse.com/novedades/certificado-ambiental-earth-check-mexico-148216.html. 
Bohdanowicz, P. (2005). European hoteliers' environmental attitudes: Greening the business. Cornell Hotel and Restaurant Administration Quarterly, 46(2), 188-204.

Briassoulis, H. (2002). Sustainable tourism and the question of the commons. Annals of Tourism Research, 29, 1065-1085.

Carmona, E., Céspedes, J., \& De Burgos, J. (2004). Environmental strategies in Spanish hotels: Contextual factors and performance. The Service Industries Journal, 24(3), 101-130.

Céspedes, J. y De Burgos, J. (2004). Un análisis de las dimensiones de la gestión ambiental en los servicios hoteleros. Revista de Ingeniería de Organización, 30, 119-237.

Chan, W., \& Lam, J. (2003). Energy-saving supporting tourism sustainability: A case study of hotel swimming pool heat pump. Journal of Sustainable Tourism, 11(1), 74-83.

Christmann, P. (2000). Effects of best practices of environmental management on cost advantage: The role of complementary assets. Academy of Management Journal, 43(4), 663-680.

Claver, E., Molina, J., Pereira, J., \& López, M. (2007a). Environmental strategies and their impact on hotel performance. Journal of Sustainable Tourism, 15(6), 663-679.

Claver, E., López, M., Molina, J., \& Tarí, J. (2007b). Environmental management and firm performance: A case study. Journal of Environmental Management, 84(4), 606-619.

Claver-Cortés, E., Pereira-Moliner, J., Tarí, J. J., \& Molina-Azorín, J. F. (2008). TQM, managerial factors and performance in the Spanish hotel industry. Industrial Management \& Data Systems, 108(2), 228-244.

Cramer, J. (1998). Environmental management: From fit to stretch. Business Strategy and the Environment, $7(3), 162-172$.

De Burgos, J. y Céspedes, J. (2001). La protección ambiental y el resultado: un análisis crítico de su relación. Investigaciones Europeas de Dirección y Economía de la Empresa, 7(2), 93-108.

De Burgos, J., Vázquez, D., Plaza, J., \& Dijkshoorn, J. (2013). Environmental protection and financial performance: An empirical analysis in Wales. International Journal of Operations and Production Management, 33(8), 981-1018.

de Yta-Castillo, D. y Sánchez-Medina, P. S. (2017). Estrategias ambientales y desempeño empresarial de hoteles de México. Temas de Ciencia y Tecnología, 21(63), 5-21.

EarthCheck Certificación (2016). Productos y servicios. Benchmarking and certification. Disponible en https:// es.earthcheck.org/products-services/certificacion/benchmarking-and-certification/

El Dief, M., \& Font, X. (2010). Determinants of environmental management in the red sea hotels: Personal and organizational values and contextual variables. International Centre for Responsible Tourism, $36(1), 115-137$.

Enz, C. A., \& Siguaw, J. A. (1999). Best hotel environmental practices. Cornell Hotel and Restaurant Administration Quarterly, 40(5), 72-77. Disponible en http://scholarship.sha.cornell.edu/articles/488/

Erdogan, N., \& Baris, E. (2007). Environmental protection programs and conservation practices of hotels in Ankara. Turkey. Tourism Management, 28(2), 604-614.

Fraj, E., Martínez, E., \& Matute, J. (2009). A multidimensional approach to the influence of environmental marketing and orientation on the firm's organizational performance. Journal of Business Ethics, $88(2), 263-286$.

Fraj, E., Matute, J., \& Melero, I. (2015). Environmental strategies and organizational competitiveness in the hotel industry: The role of learning and innovation as determinants of environmental success. Tourism Management, 46, 30-42.

Fukey, L., \& Issac, S. (2014). Connect among green, sustainability and hotel industry: A prospective simulation study. International Journal of Social, Management, Economics and Business Engineering, 8, 311-327.

Galdeano, E., Céspedes, J., \& Del Río, J. (2008). Environmental performance and spillover effects on productivity: Evidence from horticultural firms. Journal of Environmental Management, 88(4), 1552-1561. 
Galindo, L. M. (2009). La economía del cambio climático en México. Síntesis. Secretaría de Hacienda y Crédito Público y Secretaría de Medio Ambiente y Recursos Naturales.

Garay, L., \& Font, X. (2012). Doing good to do well? Corporate social responsibility reasons, practices and impacts in small and medium accommodation enterprises. International Journal of Hospitality Management, 31(2), 329-337.

García, F., \& Armas, Y. (2007). Relation between social-environmental responsibility and performance in hotel firms. International Journal of Hospitality Management, 26(3), 824-839.

Hardin, G. (1968). The tragedy of the commons. Science, 162(3859), 1243-1248.

Hart, S. (1995). A natural resource based view of the firm. Academy of Management Review, 20(4), 986-1014.

Hart, S. (1997). Beyond greening: Strategies for a sustainable world. Harvard Business Review, 75(1), 67-76.

Hart, S., \& Milstein, M. (2003). Creating sustainable value. Academy of Management Executive, 17(2), 56-67.

Henri, J., \& Journeault, M. (2008). Environmental performance indicators: An empirical study of Canadian manufacturing firms. Journal of Environmental Management, 87(1), 165-176.

Hernández, R., Fernández-Collado, C. y Baptista, P. (2010). Metodología de la investigación. México: McGraw-Hill.

Hutchinson, C. (1996). Integrating environment policy with business strategy. Long Range Planning, 29(1), 11-23.

Instituto Mexicano para la Competitividad A. C. (2013). Nueva política turística para recuperar la competitividad del sector y detonar el desarrollo regional. Instituto Mexicano para la Competitividad A. C. Disponible en http://imco.org.mx/wp-content/uploads/2013/10/Turismo2013_Completo.pdf

Jones, P., Hillier, D., \& Comfort, D. (2014). Assurance of the leading UK food retailers' corporate social responsibility/sustainability reports. Corporate Governance, 14(1), 130-138.

Johnstone, D., \& Labonne, J. (2009). Why do manufacturing facilities introduce environmental management systems? Improving and/or signaling performance. Ecological Economics, 68(3), 719-730.

Kasim, A. (2007). Towards a wider adoption of environmental responsibility in the hotel sector. International Journal of Hospitality \& Tourism Administration, 8(2), 25-49.

Kasim, A., Gursoy, D., Okumus, F., \& Wong, A. (2014). The importance of water management in hotels: A framework for sustainability through innovation. Journal of Sustainable Tourism, 22(7), 1090-1107.

Kirk, D. (1995). Environmental management in hotels. International Journal of Contemporary Hospitality Management, 7(6), 3-8.

Klassen, R., \& McLaughlin, C. (1996). The impact of environmental management on firm performance. Journal of Management Science, 42(8), 1199-1214.

Klassen, R., \& Whybark, C. (1999). Environmental management in operations: The selection of environmental technologies. Decision Sciences, 30(3), 601-631.

Klassen, R., \& Mclaughlin, C. (2001). The impact of environmental management on firm performance. Management Science, 42(8), 1200-1213.

Kumar, P. (2015). Green marketing innovations in small Indian firms. World Journal of Entrepreneurship, Management and Sustainable Development, 11(3), 176-190.

Le, Y., Hollenhorst, S., Harris, C., McLaughlin, W., \& Shook, S. (2006). Environmental management: A study of Vietnamese hotels. Annals of Tourism Research, 33(2), 545-567.

Leonidou, L. C., Leonidou, C. N., Fotiadis, T. A., \& Zeritini, A. (2013). Resources and capabilities as drivers of hotel environmental marketing strategy: Implications for competitive advantage and performance. Tourism Management, 35, 94-110.

López-Gamero, M., Claver-Cortés, E., \& Molina-Azorín, J. (2008). Complementary resources and capability for an ethical and environmental management: A qual/quan study. Journal of Business Ethics, 82(3), 701-732. 
López-Gamero, M., Molina-Azorín, J., \& Claver-Cortés, E. (2009). The whole relationship between environmental variables and firm performance: Competitive advantage and firm resources as mediator variables. Journal of Environmental Management, 90(10), 3110-3121.

López-Gamero, M. D., Molina-Azorín, J., \& Claver-Cortés, E. (2010). The potential of environmental regulation to change managerial perception, environmental management, competitiveness and financial performance. Journal of Cleaner Production, 18(10), 963-974.

Luja, V. H. y Saldaña, C. E. (2014). Amor y odio: efectos ambientales, económicos y sociales del turismo. Estados Unidos de América. Universidad Autónoma de Nayarit.

Maas, S., Schuster, T., \& Hartmann, E. (2014). Pollution prevention and service stewardship strategies in the third-party logistics industry: Effects on firm differentiation and the moderating role of environmental communication. Business Strategy and the Environment, 23, 38-55.

Menguc, B., Auh, S., \& Ozanne, L. (2010). The interactive effect of internal and external factors on a proactive environmental strategy and its influence on a firm's performance. Journal of Business Ethics, 94(2), 279-298.

Menon, A., \& Menon, A. (1997). Enviropreneurial marketing strategy: The emergence of corporate environmentalism as market strategy. Journal of Marketing, 61, 51-67.

Molina-Azorín, J. F., Claver-Cortés, E., Pereira-Moliner, J. \& Tarí, J. J. (2009a). Environmental practices and firm performance: An empirical analysis in the Spanish hotel industry. Journal of Cleaner Production, 17, 516-524.

Molina-Azorín, J. F., Tarí, J. J., Claver-Cortés, E., \& López-Gamero, M. D. (2009b). Quality management, environmental management and firm performance: A review of empirical studies and issues of integration. International Journal of Management Reviews, 11(2), 197-222.

Molina-Azorín, J. F., Tarí, J. J., Pereira-Moliner, J., López-Gamero, M. D., \& Pertusa-Ortega, E. M. (2015). The effects of quality and environmental management on competitive advantage: A mixed methods study in the hotel industry. Tourism Management, 50, 41-54.

Montabon, F., Sroufe, R., \& Narasimhan, R. (2007). An examination of corporate reporting: environmental management practices and firm performance. Journal of Operations Management, 25(5), 998-1014.

Muñoz, C., Rivera, M., \& Oliveras, C. (2005). Turismo y conciencia ambiental en México. Gaceta Ecológica, 75, 5-18. Organización Mundial del Turismo (OMT) (2008). Climate change and tourism. Responding to global challenges. Madrid: Organización Mundial del Turismo.

Organización Mundial del Turismo (OMT) (2010). Tourism and the millennium development goals. Madrid: Organización Mundial del Turismo.

Organización Mundial del Turismo (OMT) (2016). Panorama OMT del turismo internacional.

Oreja, J., \& Armas, Y. (2012). Environmental performance in the hotel sector: The case of the Western Canary Islands. Journal of Cleaner Production, 29, 64-72.

Park, J., \& Kim, H. (2014). Environmental proactivity of hotel operations: Antecedents and the moderating effect of ownership type. International Journal of Hospitality Management, 37, 1-10.

Pereira-Moliner, J., Claver-Cortés, E., Molina-Azorín, J. F., \& Tarí, J. J. (2012). Quality management, environmental management and firm performance: Direct and mediating effects in the hotel industry. Journal of Cleaner Production, 37, 82-92.

Porter, M., \& Van der Linde, C. (1995). Green and competitive: Ending the stalemate. Harvard Business Review, 73(5), 120-134.

Rivera, J. (2002). Assessing a voluntary environmental initiative in the developing world: the Costa Rican certification for sustainable tourism. Policy Sciences, 35, 333-360.

Russo, M. V., \& Fouts, P. A. (1997). A resource-based perspective on corporate environmental performance and profitability. Academy of Management Journal, 40(3), 534-559. 
Sánchez-Medina, P. S., Díaz-Pichardo, R., \& Cruz-Bautista, M. (2016). Stakeholder influence on the implementation of environmental management practices in the hotel industry. International Journal of Tourism Research, 18(4), 387-398.

Sarkis, J., González, P., \& Adenso, B. (2010). Stakeholder pressure and the adoption of environmental practices: the mediating effect of training. Journal of Operations Management, 28(2), 163-176.

Sharma, S., \& Vredenburg, H. (1998). Proactive corporate environmental strategy and the development of competitively valuable organizational capabilities. Strategic Management Journal, 19, 729-753.

Song, H., Dwyer, L., Li, G., \& Cao, Z. (2012). Tourism economics research: A review and assessment. Annals of Tourism Research, 39(3), 1653-1682.

Shrivastava, P. (1995). Environmental technologies and competitive advantage. Strategic Management Journal, $16,183-200$.

Sroufe, R. (2003). Effects of environmental management systems on environmental management practices and operations. Product and Operations Management, 12(3), 416-432.

Tarí, J. J., Claver-Cortés, Pereira-Moliner, J., \& Molina-Azorín, J. F. (2010). Levels of quality and environmental management in the hotel industry: Their joint influence on firm performance. International Journal of Hospitality Management, 29, 500-510.

Teng, C. C., Horng, J. S., Hu, M. L., Chien, L. H., \& y Shen, Y. C. (2012). Developing energy conservation and carbon reduction indicators for the hotel industry in Taiwan. International Journal of Hospitality Management, 31(1), 199-208.

Vargas, E., Zizumbo, L., Viesca, F., y Serrano, R. (2011). Gestión ambiental en el sector turístico mexicano. Efectos de la regulación en el desempeño hotelero. Cuadernos de Administración, 24(42), 183-204.

Vargas, E. E. (2015). Responsabilidad social empresarial y gestión ambiental en el sector hotelero. Toluca: Universidad Autónoma del Estado de México.

Venkatraman, N., \& Ramanujam, V. (1986). Measurement of business performance in strategy research: A comparison of approaches. Academy of Management Review, 11(4), 801-814.

Wahba, H. (2008). Does the market value corporate environmental responsibility? An empirical examination. Corporate Social Responsibility and Environmental Management, 15(2), 89-99.

Wagner, M., Van Phu, N., Azomahou, T., \& Wehrmeyer, W. (2002). The relationship between the environmental and economic performance of firms: An empirical analysis of the European paper industry. Corporate Social Responsibility and Environmental Management, 9, 133-146.

Wagner, M. (2005). Sustainability and competitive advantage: Empirical evidence on the influence of strategic choices between environmental management approaches. Environmental Quality Management, 14(3), 31-48.

Walsh, P., \& Dodds, R. (2017). Measuring the choice of environmental sustainability strategies in creating a competitive advantage. Business Strategy and the Environment. http://10.1002/bse.1949

Xuchao, W., Priyadarsini, R., \& Eang, L. (2010). Benchmarking energy use and green house gas emissions in Singapore's hotel industry. Energy Policy, 38(8), 4520-4527.

Yang, M. G., Hong, P., \& Modi, S. B. (2011). Impact of lean manufacturing and environmental management on business performance: An empirical study of manufacturing firms, International Journal of Production Economics, 129, 251-261.

Zhu, Q., \& Sarkis, J. (2004). Relationships between operational practices and performance among early adopters of green supply chain management practices in Chinese manufacturing enterprises. Journal of Operations Management, 22(3), 265-289.

\section{BY-NC-ND}

